University of Warwick institutional repository: http://go.warwick.ac.uk/wrap This paper is made available online in accordance with publisher policies. Please scroll down to view the document itself. Please refer to the repository record for this item and our policy information available from the repository home page for further information.

To see the final version of this paper please visit the publisher's website. Access to the published version may require a subscription.

Author(s): THOMAS JORDAN and MARK POLLICOTT

Article Title: Properties of measures supported on fat Sierpinski carpets Year of publication: 2006

Link to published version: http://dx.doi.org/

10.1017/S0143385705000696

Publisher statement: None 


\title{
Properties of measures supported on fat Sierpinski carpets
}

\author{
THOMAS JORDAN and MARK POLLICOTT \\ Department of Mathematics, Warwick University, Coventry CV4 7AL, UK \\ (e-mail: \{tjordan, mpollic\}@maths.warwick.ac.uk)
}

(Received 23 June 2004 and accepted in revised form 29 November 2004)

\begin{abstract}
In this paper we study certain conformal iterated function schemes in two dimensions that are natural generalizations of the Sierpinski carpet construction. In particular, we consider scaling factors for which the open set condition fails. For such 'fat Sierpinski carpets' we study the range of parameters for which the dimension of the set is exactly known, or for which the set has positive measure.
\end{abstract}

\section{$0 . \quad$ Introduction}

In this paper we want to study a simple conformal iterated function scheme that fails to satisfy the standard open set condition. Let $0<\lambda<1$. Given $n>k$ we want to consider a family of $n$ conformal contractions $T_{i}: \mathbb{R}^{2} \rightarrow \mathbb{R}^{2}$ of the form

$$
T_{i}:(x, y) \mapsto(\lambda x, \lambda y)+\left(c_{i}^{(1)}, c_{i}^{(2)}\right),
$$

$i=1, \ldots, n$, where $\left(c_{i}^{(1)}, c_{i}^{(2)}\right) \in\left\{(j, l) \in \mathbb{Z}^{2}: 0 \leq j, l \leq k-1\right\}$ are $n$ distinct points in a $k \times k$ grid. There is then a unique smallest closed set $\Lambda_{\lambda}$ such that $\Lambda_{\lambda}=\bigcup_{i=1}^{k} T_{i}\left(\Lambda_{\lambda}\right)$. In the special case that $\lambda=1 / k$, the sets $\Lambda_{1 / k}$ are the well-known Sierpinski carpets. If $\lambda \in(0,1 / k)$ then the contractions satisfy the open set condition and $\Lambda_{\lambda}$ is a Cantor set whose dimension we can easily compute as

$$
\operatorname{dim}_{H}\left(\Lambda_{\lambda}\right)=-\frac{\log n}{\log \lambda}
$$

In this paper we shall extend this equality to a strictly larger parameter set of $\lambda$. Unfortunately, we cannot expect this identity to hold on a larger interval since it is easy to see that there are examples with a countable dense set of exceptional values $\mathcal{E} \subset[1 / k, 1 / \sqrt{n}]$ such that $(0.1)$ fails for $\lambda \in \mathcal{E}$. Our first result extends these results to a larger set, as shown by Figure 1 .

THEOREM 1. There exists $1 / k \leq s \leq 1 / \sqrt{n}$ such that for almost all $\lambda \in(1 / k, s]$ we have $\operatorname{dim}_{H}\left(\Lambda_{\lambda}\right)$ is given by $(0.1)$. 


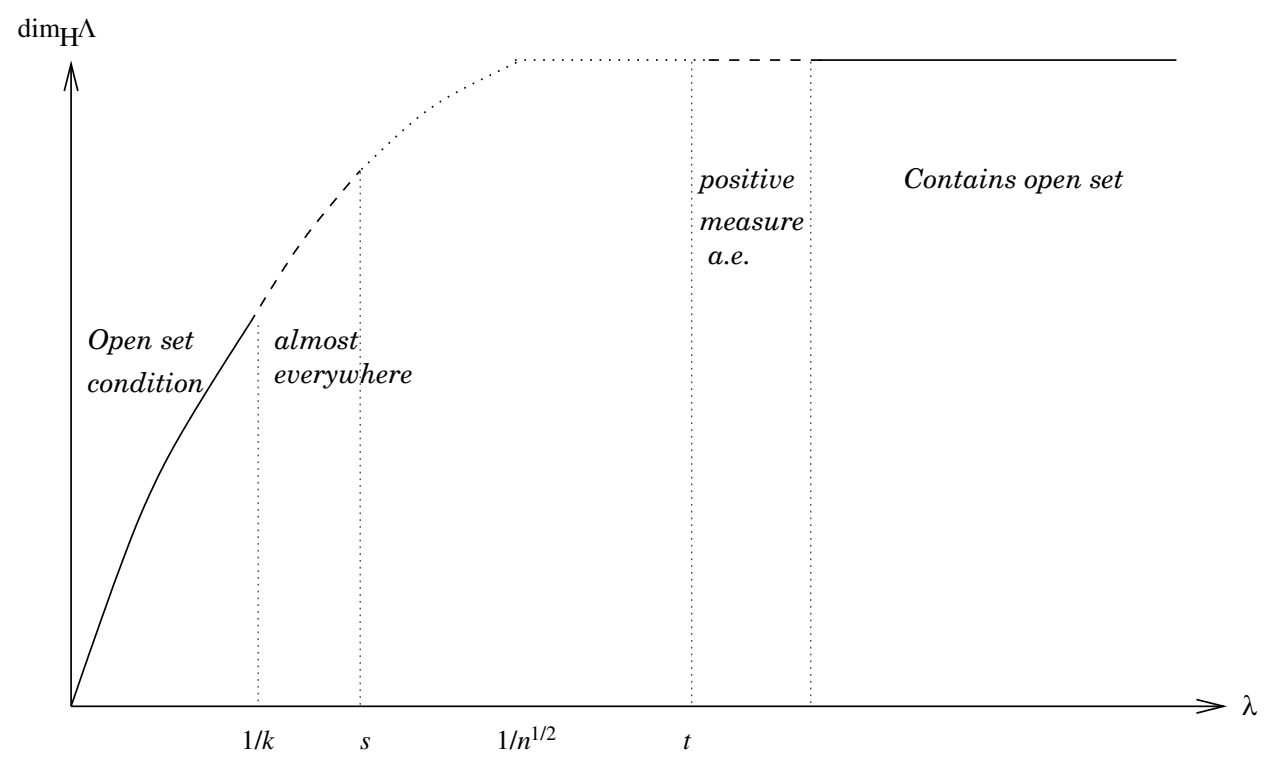

FIGURE 1. Extending the regions where dimension is known.

We can give an explicit estimate for $s$. More precisely, we denote the number of images in the $j$ th row by $n_{j}=\operatorname{Card}\left\{1 \leq l \leq k: c_{i}^{(1)}=j\right\}$, for $j=1, \ldots, k$. If we assume that $n_{i} \geq 1$, then we can take

$$
s=\min \left\{\frac{1}{n}\left(\prod_{j=1}^{k} n_{j}^{n_{j}}\right)^{1 / n},\left(\prod_{j=1}^{k} n_{j}^{-n_{j}}\right)^{1 / n}\right\} .
$$

The following is a simple corollary to Theorem 1.

COROLlaRY. There exists a dense $G_{\delta}$ set $\mathcal{G} \subset[1 / k, s]$ such that for $\lambda \in \mathcal{G}, \operatorname{dim}_{\mathrm{H}}\left(\Lambda_{\lambda}\right)$ is given by (0.1).

Proof. This follows from the semi-continuity of the map $\lambda \mapsto \operatorname{dim}_{H}\left(\Lambda_{\lambda}\right)[6,17]$.

Providing $\lambda$ is sufficiently large, we might expect the set to have positive measure. An ingredient in the study of this problem is a development of the idea of transversality [14]. This leads to a technical constraint in proving these theorems which requires that $t \leq b_{k-1}$, where $b_{k-1}$ is a transversality constant. For example, $b_{1}=0.649 \ldots$ and $b_{2}=0.5$.

THEOREM 2. There exists $1 / \sqrt{n} \leq t \leq b_{k-1}$ such that for almost all $\lambda \in\left[t, b_{k-1}\right]$ we have that $\operatorname{leb}\left(\Lambda_{\lambda}\right)>0$.

We can give an explicit estimate for $t=t\left(n_{1}, \ldots, n_{k}\right)$ :

$$
t=\sup \left\{\prod_{j=1}^{k} q_{j}^{n_{j}}: \sum_{j=1}^{k} q_{j} \log \left(\frac{q_{j}}{n_{j}}\right)=0, \sum_{j=1}^{k} q_{j}=1 \text { and } q_{j} \geq 0\right\} .
$$



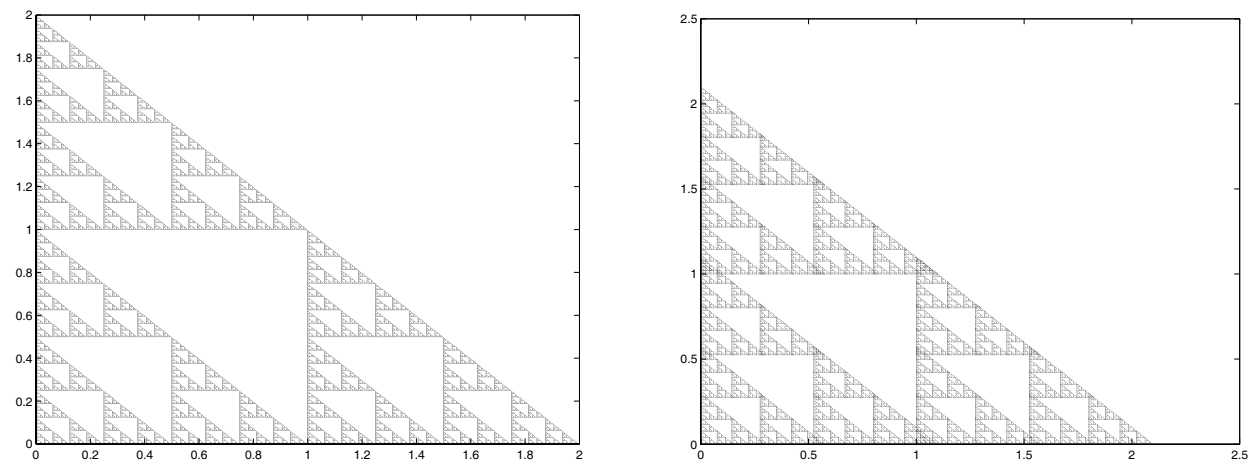

FIGURE 2. Sierpinski gasket where (i) $\lambda=0.5$ and (ii) $\lambda=0.525$.
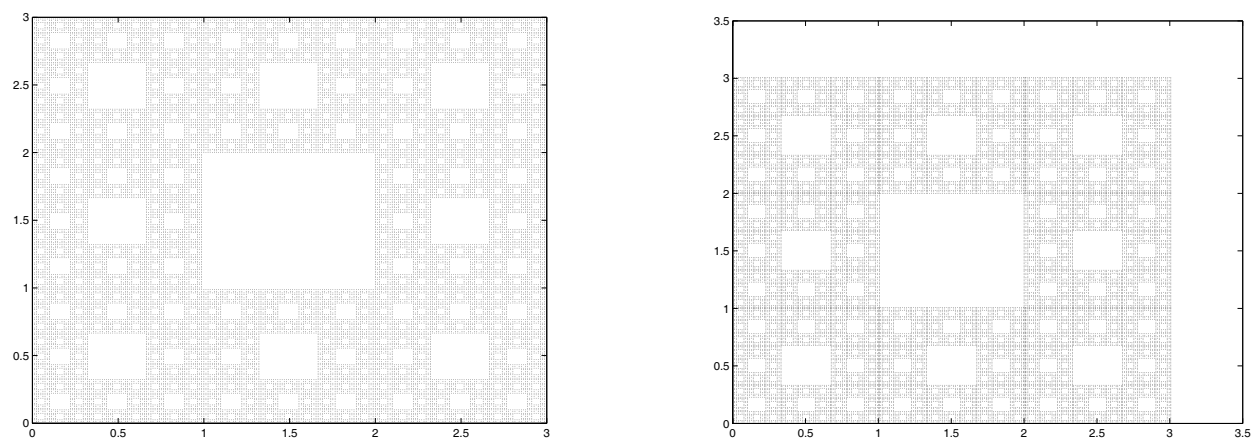

FIGURE 3. Sierpinski carpets where (i) $\lambda=1 / 3$ and (ii) $\lambda=0.338$.

Example 1. (The Sierpinski triangle (Figure 2)) Let $k=2$ and $c_{1}=(0,0), c_{2}=(1,0)$ and $c_{3}=(0,1)$. Broomhead et al [4] computed the dimension of $\Lambda_{\lambda}$ at certain exceptional values $\omega_{n} \searrow 0$, called multinacci numbers, characterized as roots of $3 x^{n+1}-3 x+1$ (e.g., $\omega_{2}=0.618 \ldots, \omega_{3}=0.543 \ldots, \omega_{4}=0.518 \ldots$, etc.).

Jordan [7] established Theorem 1 with $s=2^{2 / 3} / 3=0.529 \ldots$ Theorem 2 applies with $t=0.5852 \ldots$ (corresponding to the choice $q_{2}=0.7729 \ldots$ ). For comparison, in [4] it is shown that for $\lambda \geq 0.647 \ldots$ the set $\Lambda_{\lambda}$ contains open sets.

Example 2. (The Sierpinski carpet (Figure 3)) Let $k=3$ and $c_{1}, \ldots, c_{8}$ are all but the central square. In Theorem 1, we can take $s=\left(3^{3} 2^{3} 3^{3}\right)^{1 / 8} / 8=0.338851 \ldots$ In Theorem 2, we can take $t=0.357 \ldots$ (corresponding to the choices $q_{1}=q_{3}=$ $0.416 \ldots$ and $\left.q_{2}=0.168 \ldots\right) \dagger$.

Example 3. (Vicsek set (Figure 4)) Let $k=3$ and let $c_{1}, \ldots, c_{5}$ correspond to a cross. In this case $s=\left(3^{3}\right)^{1 / 5} / 5=0.386636 \ldots$ and $t=0.4541$.

$\dagger$ One trivially sees that one could choose $t=1 / 2$, since for $\lambda \geq 1 / 2$ we have that $\Lambda_{\lambda}$ is a square, and thus has positive measure. 

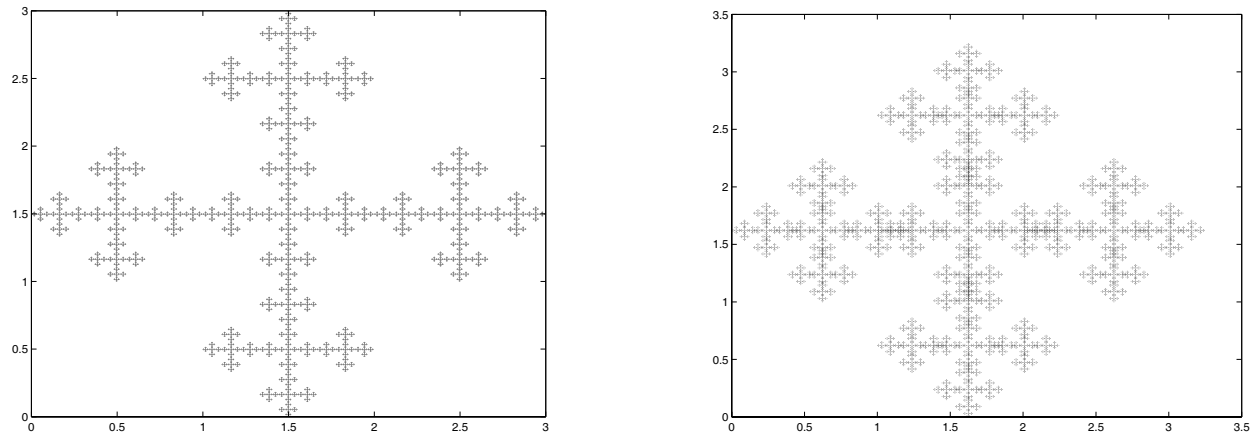

FIGURE 4. Vicsek sets where (i) $\lambda=1 / 3$ and (ii) $\lambda=0.386$.

TABLE 1. Summary of examples.

\begin{tabular}{llccc}
\hline Shape & \multicolumn{1}{c}{$1 k$} & \multicolumn{1}{c}{$s$} & $1 / \sqrt{n}$ & $t$ \\
\hline Triangle & 0.5 & $0.529 \ldots$ & $0.577 \ldots$ & $0.585 \ldots$ \\
Carpet & $0.333 \ldots$ & $0.338 \ldots$ & $0.353 \ldots$ & $0.357 \ldots$ \\
Cross & $0.333 \ldots$ & $0.386 \ldots$ & $0.447 \ldots$ & $0.454 \ldots$ \\
\hline
\end{tabular}

These examples are summarized in Table 1.

In $\S 1$ we formulate a general result on projections of measures. In $\S \S 2$ and 3 we develop the technical results. The proof of Theorem 1 is completed in $\S 3$. In $\S \S 4$ and 5 we give the proof of Theorem 2. In $\S 6$, we consider generalizations.

\section{Subshifts and Invariant measures}

Let $\Sigma_{n}=\{1,2, \ldots, n\}^{\mathbb{Z}^{+}}$be the space of sequences and let $\sigma: \Sigma_{n} \rightarrow \Sigma_{n}$ be the full shift on $n$ symbols defined by $(\sigma x)_{n}=x_{n+1}$. Let $\Pi_{\lambda}: \Sigma_{n} \rightarrow \mathbb{R}^{2}$ be defined by

$$
\Pi_{\lambda}\left(x_{m}\right)=\sum_{m=0}^{\infty} c_{x_{m}} \lambda^{m}
$$

The (two-dimensional) fat Sierpinski carpet $\Lambda_{\lambda} \subset \mathbb{R}^{2}$ is defined by

$$
\Lambda_{\lambda}=\left\{\Pi_{\lambda}(x)=\sum_{m=0}^{\infty} c_{x_{m}} \lambda^{m}: x=\left(x_{m}\right)_{m=0}^{\infty} \in \Sigma_{n}\right\} \text {. }
$$

Let $\Sigma_{k}=\{1,2, \ldots, k\}^{\mathbb{Z}^{+}}$. We can define a factor map $p: \Sigma_{n} \rightarrow \Sigma_{k}$ by $(p(x))_{i}=$ $c_{x_{i}}^{(1)}$ where $c_{x_{i}}=\left(c_{x_{i}}^{(1)}, c_{x_{i}}^{(2)}\right)$, for $i \in \mathbb{Z}^{+}$. Let $\bar{\Pi}_{\lambda}: \Sigma_{k} \rightarrow \mathbb{R}$ be defined by $\bar{\Pi}_{\lambda}\left(y_{m}\right)=$ $\sum_{m=0}^{\infty} y_{m} \lambda^{m}$. We can associate a closed set $\Lambda_{\lambda} \subset \mathbb{R}$ to $\Sigma_{k}$ defined by

$$
\bar{\Lambda}_{\lambda}=\left\{\bar{\Pi}_{\lambda}(y)=\sum_{m=0}^{\infty} y_{m} \lambda^{m}: y=\left(y_{m}\right)_{m=0}^{\infty} \in \Sigma_{k}\right\} .
$$

Let $\pi: \mathbb{R}^{2} \rightarrow \mathbb{R}$ be the horizontal projection $\pi(x, y)=y$ on the vertical axis. Then we can write $\bar{\Pi}_{\lambda} \circ p=\pi \circ \Pi_{\lambda}$. 
Let $\mu$ be an ergodic shift invariant probability measure on $\Sigma_{n}$. The image $\bar{\mu}:=p(\mu)$ of $\mu$ under $p: \Sigma_{n} \rightarrow \Sigma_{k}$ is defined by $\bar{\mu}(A)=\mu\left(p^{-1} A\right)$, where $A \subset \Sigma_{k}$ is Borel. The probability measure $\bar{\mu}$ is an ergodic shift invariant probability measure on $\Sigma_{k}$.

The measure $\mu$ projects to a measure $v_{\lambda}=\Pi_{\lambda}(\mu)$ on $\Lambda_{\lambda}$. The Hausdorff dimension $\operatorname{dim}_{H}\left(\nu_{\lambda}\right)$ of $\nu_{\lambda}$ is defined to be the infimum of the Hausdorff dimension of Borel sets of full $v_{\lambda}$-measure. The projection $\bar{v}_{\lambda}=\pi\left(v_{\lambda}\right)$ of the measure defined by $\bar{v}_{\lambda}(B)=v_{\lambda}(B \times \mathbb{R})$, where $B \subset \mathbb{R}$ is a Borel subset. We can also write $\bar{\nu}_{\lambda}=\bar{\Pi}_{\lambda}(\bar{\mu})$.

Let $h(\mu)$ denote the entropy of $\sigma:\left(\Sigma_{n}, \mu\right) \rightarrow\left(\Sigma_{n}, \mu\right)$ and let $h(\bar{\mu})$ denote the entropy of $\sigma:\left(\Sigma_{k}, \bar{\mu}\right) \rightarrow\left(\Sigma_{k}, \bar{\mu}\right)$ (see [20]). Our main technical result is the following.

THEOREM 3. For almost all $\lambda \in[1 / k, 1 / \sqrt{n}]$ we have that:

$$
\begin{gathered}
\operatorname{dim}_{H}\left(v_{\lambda}\right)=-\frac{h(\mu)}{\log \lambda} \quad \text { if } \max \left\{-\frac{h(\bar{\mu})}{\log \lambda},-\frac{h(\mu)-h(\bar{\mu})}{\log \lambda}\right\} \leq 1 ; \\
\operatorname{dim}_{H}\left(v_{\lambda}\right) \in\left[\min \left\{1-\frac{h(\bar{\mu})}{\log \lambda}, 1-\frac{h(\mu)-h(\bar{\mu})}{\log \lambda}\right\},-\frac{h(\mu)}{\log \lambda}\right] \text { otherwise. }
\end{gathered}
$$

Remark. Assume that there are two adjacent squares in the carpet. It is easy to show that there is a dense set of values $\mathcal{E} \subset[1 / k, 1 / \sqrt{n}]$ such that for $\lambda \in \mathcal{E}$ we have $\operatorname{dim}_{H}\left(\Lambda_{\lambda}\right)<-\log n / \log \lambda$. More precisely, for $N$ suitably large, appropriately small changes in $\lambda$ can cause two $N$ th level squares (of size $\lambda^{N}$ ) to coincide. This results in a drop in the dimension. This suffices to show that $\operatorname{dim}_{H}\left(\nu_{\lambda}\right)<-h(\mu) / \log \lambda$ for any fully supported measure (cf. [17]).

\section{Hausdorff dimension, projections and transversality}

In this section we recall some definitions and basic properties. Given $\delta, \epsilon>0$ we can define

$$
H_{\epsilon}^{\delta}(\Lambda)=\inf _{\left\{U_{i}\right\}}\left\{\sum_{i}\left(\operatorname{diam}\left(U_{i}\right)\right)^{\delta}\right\},
$$

where the infimum is over all covers $\left\{U_{i}\right\}$ for $\Lambda$ where $\sup _{i}\left\{\operatorname{diam}\left(U_{i}\right)\right\} \leq \epsilon$. The $\delta$-dimensional Hausdorff dimension of $\Lambda$ is defined by $H^{\delta}(\Lambda)=\lim _{\epsilon}{ }_{0} H_{\epsilon}^{\delta}(\Lambda)$. Finally, the Hausdorff dimension of $\Lambda$ is defined by

$$
\operatorname{dim}_{H}(\Lambda)=\inf \left\{H^{\delta}(\Lambda)=0\right\}
$$

A key technical device is transversality. This was first introduced in [14], but subsequently refined and developed by Peres, Solomyak and others $[\mathbf{1 1}, \mathbf{1 8}]$. The following version is useful in the sequel.

Proposition 2.1. [11] Given $k \geq 2$ and $0<s<1$ there exists $b_{k}>1 / k$ and $K=K(s)>0$ such that for

(i) any sequence $a_{n} \in\{-k, \ldots, k\}, n \geq 1$ and

(ii) any $a_{0} \in\{-k, \ldots, k\}-\{0\}$,

we have that

$$
\left|\int_{0}^{b_{k}} \frac{d \lambda}{\left|a_{0}+\sum_{n=1}^{\infty} a_{n} \lambda^{n}\right|^{s}}\right| \leq K
$$


The first few values of $b_{n}$ can be estimated numerically [11]: $b_{1}=0.649 \ldots, b_{2}=0.5$, $b_{3}=0.427 \ldots, b_{4}=0.371 \ldots, b_{5}=0.325 \ldots$ and afterwards $b_{n}=(1+\sqrt{n})^{-1}$.

The dimension of the one-dimensional measure $\bar{v}$ has been studied by Simon, Solomyak and Urbanski, who showed the following result.

Proposition 2.2. [18] For almost all $0<\lambda<b_{k-1}$ we have that

$$
\operatorname{dim}_{H}(\bar{\nu})=\min \left\{1,-\frac{h(\bar{\mu})}{\log \lambda}\right\} .
$$

\section{Conditional entropy}

We begin by recalling a few basic properties of the entropy of an invariant measure $\mu$. Let $\alpha=\{[0],[1], \ldots,[n]\}$ be the standard generating partition for $\Sigma_{n}$. Given $N \geq 1$, we can associate $x \in \Sigma_{n}$ to a cylinder

$$
\left[x_{0}, \ldots, x_{N-1}\right]=\left\{y \in \Sigma_{n}: y_{j}=x_{j}, 0 \leq j \leq N-1\right\}
$$

of length $N$. We denote by $\bigvee_{i=0}^{N-1} \alpha$ the partition consisting of all such cylinders and entropy $H_{\mu}\left(\bigvee_{i=0}^{N-1} \alpha\right)$. The entropy $h(\mu)$ of $\sigma:\left(\Sigma_{n}, \mu\right) \rightarrow\left(\Sigma_{n}, \mu\right)$ is defined by

$$
h(\mu)=\lim _{n \rightarrow+\infty} \frac{1}{N} H_{\mu}\left(\bigvee_{i=0}^{N-1} \alpha\right) .
$$

The asymptotic measure of a cylinder is given by the Shannon-McMillan-Brieman theorem, i.e. for a.e. $(\mu) x \in \Sigma_{n}$,

$$
h(\mu)=-\lim _{N \rightarrow+\infty} \frac{1}{N} \log \mu\left(\left[x_{0}, \ldots, x_{N-1}\right]\right)
$$

(see [20] and [12, p. 261]).

Let $\mathcal{B}\left(\Sigma_{n}\right)$ be the Borel sigma algebra for $\Sigma_{n}$, and let $\mathcal{B}\left(\Sigma_{k}\right)$ be the Borel sigma algebra for $\Sigma_{k}$. Let $\mathcal{A}=p^{-1} \mathcal{B}\left(\Sigma_{k}\right) \subset \mathcal{B}\left(\Sigma_{n}\right)$ be the corresponding $\sigma$-invariant sub-sigma algebra (i.e. the sigma algebra which does not distinguish between the symbols in $\{1, \ldots, n\}$ which project to the same symbol in $\{1, \ldots, k\})$.

We let $H_{\mu}(P \mid \mathcal{C})$ denote conditional entropy of a partition $P$, with respect to a sigma algebra $\mathcal{C}$.

Notation. The conditional entropy of $\sigma:\left(\Sigma_{n}, \mu\right) \rightarrow\left(\Sigma_{n}, \mu\right)$ with respect to $\mathcal{A}$ is given by

$$
h(\mu \mid \mathcal{A})=\lim _{N \rightarrow+\infty} \frac{1}{N} H_{\mu}\left(\bigvee_{i=0}^{N-1} \alpha \mid \mathcal{A}\right)
$$

(see [1], [13], [9] and [15]). In particular, $h(\mu \mid \mathcal{A}) \leq h(\mu)$.

We can uniquely decompose the probability measure $\mu$ by

$$
\mu(A)=\int \mu_{\xi}\left(p^{-1} \xi \cap A\right) d \bar{\mu}(\xi)
$$

for any Borel set $A \subset \Sigma_{n}$ (see $\left.[16, \S 1.7]\right)$, where we denote by $\mu_{\xi}$ the conditional probability measures on the fibres $p^{-1}(\xi)\left(\xi \in \Sigma_{k}\right)$. A set $X \subset \Sigma_{n}$ satisfies $\mu(X)=1$ 
precisely when there is a set $Y \subset \Sigma_{k}$ with $\bar{\mu}(Y)=1$ such that $\mu_{\xi}\left(p^{-1} \xi \cap X\right)=1$, for all $\xi \in Y$. The following result can be viewed as an analogue of the Shannon-McMillanBrieman theorem on the fibres $p^{-1}(\xi)$, and it appears in the work of Ledrappier and Young [8].

Proposition 3.1. (cf. [8]) For a.e. $(\mu) x \in \Sigma_{n}$,

$$
\lim _{N \rightarrow 0}-\frac{\log \mu_{\xi}\left(\left[x_{0}, \ldots, x_{N-1}\right] \cap p^{-1}(\xi)\right)}{N}=h(\mu \mid \mathcal{A}) .
$$

(Equivalently, the result holds for a.e. $(\bar{\mu}) \xi \in \Sigma_{k}$ and a.e. $\left(\mu_{\xi}\right) x \in p^{-1}(\xi)$.)

Proof. We briefly recall the idea of the proof (cf. [8, Lemma 9.3.1]). We can identify $\bigvee_{i=0}^{N-1} \sigma^{-i} \alpha$ with the partition of cylinders of length $N$ and

$$
-\log \mu_{\xi}\left(\left[x_{0}, \ldots, x_{N}\right] \cap p^{-1} \xi\right)=I\left(\bigvee_{i=0}^{N-1} \sigma^{-i} \alpha \mid \mathcal{A}\right)(x),
$$

where $I(\cdot \mid \cdot)$ denotes the usual conditional information function. We see that

$$
\begin{aligned}
\frac{1}{N} I\left(\bigvee_{i=0}^{N-1} \sigma^{-i} \alpha \mid \mathcal{A}\right) & =\frac{1}{n} \sum_{k=0}^{N-1} I\left(\sigma^{-k} \alpha \mid \mathcal{A} \vee\left(\bigvee_{i=k}^{N-1} \sigma^{-i} \alpha\right)\right) \\
& =\frac{1}{n} \sum_{k=0}^{N-1} I\left(\alpha \mid T^{k} \mathcal{A} \vee\left(\bigvee_{i=1}^{N-k-1} \sigma^{-i} \alpha\right)\right) \circ \sigma^{k} \\
& \rightarrow \int I\left(\alpha \mid \mathcal{A} \vee\left(\bigvee_{i=1}^{\infty} \sigma^{-i} \alpha\right)\right) d \mu, \quad \text { a.e. }(\mu)
\end{aligned}
$$

by the Martingale theorem [12, p. 262] and using $T \mathcal{A}=\mathcal{A}$. Finally, we observe that the limit can be identified with $h(\mu \mid \mathcal{A})=H\left(\alpha \mid \mathcal{A} \vee\left(\bigvee_{i=1}^{\infty} \sigma^{-i} \alpha\right)\right)$, as required.

Let $\epsilon, \delta, \eta>0$. By Proposition 3.1 we can choose a set $X \subset \Sigma_{n}$ with $\mu(X)>1-\delta$ and $K>0$ such that for $x \in X$ we have that

$$
\mu_{\xi}\left[x_{0}, \ldots, x_{N}\right] \leq K \exp (-(h(\mu \mid \mathcal{A})-\epsilon) N), \quad \text { for } N \geq 1 .
$$

We can denote $X_{\eta}=\left\{\xi \in \Sigma_{k}: \mu_{\xi}\left(p^{-1} \xi \cap X\right) \geq 1-\eta\right\}$, then $\eta\left(1-\bar{\mu}\left(Y_{\eta}\right)\right)<\delta$, i.e. $1-\delta / \eta<\bar{\mu}\left(X_{\eta}\right)$.

Finally, we recall a classical result that relates the entropies of $\mu$ and $\bar{\mu}$.

Proposition 3.2. (Abramov-Rohlin) $h(\mu)=h(\bar{\mu})+h(\mu \mid \mathcal{A})(c f$. [1], [12, p. 256], [9] and [3]).

\section{Dimension of the induced measure on fibres}

Let $L_{y}=\{(x, y): x \in \mathbb{R}\}$ denote the horizontal line at height $y$. Given $\xi \in \Sigma_{k}$, we can use the conditional measure $\mu_{\xi}$ on $p^{-1}(\xi)$ to define a measure $v_{\lambda, \xi}$ on the line $L_{\bar{\Pi}_{\lambda}(\xi)}$ by $v_{\lambda, \xi}=\Pi_{\lambda}\left(\mu_{\xi}\right)$. Our main result in this section is the following. 
Proposition 4.1. For almost every $\lambda \in\left[1 / k, b_{k-1}\right]$ there exists a set $Y \subset \mathbb{R}$ with $\operatorname{dim}_{H}(Y)=\operatorname{dim}_{H}\left(v_{\lambda}\right)$ such that for any $\xi \in\left(\bar{\Pi}_{\lambda}\right)^{-1} Y \subset \Sigma_{k}$ we can bound

$$
\operatorname{dim}_{H}\left(v_{\lambda, \xi}\right) \geq \min \left\{-\frac{h(v \mid \mathcal{A})}{\log \lambda}, 1\right\} .
$$

Proof. It suffices to show that, given $\delta>0$, for almost all $\lambda \in\left[1 / k, b_{1}\right]$ there exists a set $X=X_{\delta} \subset \Sigma_{k}$ with $\bar{\mu}(X) \geq 1-\delta$ such that for any $\xi \in X, \operatorname{dim}\left(\nu_{\xi, \lambda}\right) \geq-h(\mu \mid \mathcal{A}) / \log \lambda$. In particular, we can take $Y=\bigcap_{n=1}^{\infty} X_{1 / n}$.

Fix $\epsilon, \epsilon^{\prime}>0$. There exists a set $X_{\epsilon^{\prime}} \subset \Sigma_{k}$ and a constant $K>0$ such that $\bar{\mu}\left(X_{\epsilon^{\prime}}\right)>1-\epsilon^{\prime}$ and for any $\underline{\xi} \in X_{\epsilon^{\prime}}$ there exists $Y_{\epsilon^{\prime}}$ such that for any $\underline{x} \in X_{\epsilon^{\prime}}$

$$
\mu_{\xi}\left[x_{0}, \ldots, x_{N}\right] \leq K \exp (-(h(\mu \mid \mathcal{A})-\epsilon) N), \quad \text { for } N \geq 1 .
$$

Denote $s=-h(\mu \mid \mathcal{A}) / \log \lambda-2 \epsilon$. We want to apply the mass distribution method with the measure $\bar{\mu}$ restricted to $X_{\epsilon^{\prime}}$ and the measure $\nu_{\lambda, \xi}$ restricted to $\Pi_{\lambda}\left(Y_{\epsilon^{\prime}}\right)$, where $\xi \in X_{\epsilon^{\prime}}$. This allows us to use the explicit bound (4.1). Consider the multiple integral

$$
I=\int_{1 / k}^{b_{k-1}} \int_{X_{\epsilon^{\prime}}}\left(\int_{\Pi_{\lambda} Y_{\epsilon^{\prime}}} \int_{\Pi_{\lambda} Y_{\epsilon^{\prime}}} \frac{d \nu_{\xi, \lambda}(x) d \nu_{\xi, \lambda}(y)}{|x-y|^{s}}\right) d \bar{\mu}(\xi) d \lambda .
$$

We want to prove finiteness by lifting $\nu_{\xi, \lambda}$ to $\mu_{\xi}$ on $p^{-1} \xi$ and then using Fubini's theorem to rewrite the integral as

$$
\begin{aligned}
I & =\int_{X_{\epsilon^{\prime}}} \int_{Y_{\epsilon^{\prime}}} \int_{Y_{\epsilon^{\prime}}} \int_{1 / k}^{b_{k-1}} \frac{d \lambda}{\left|\Pi_{\lambda}(\underline{i})-\Pi_{\lambda}(\underline{j})\right|^{s}} d \mu_{\xi}(\underline{i}) d \mu_{\xi}(\underline{j}) d \bar{\mu}(\xi) \\
& =\int_{X_{\epsilon^{\prime}}} \int_{Y_{\epsilon^{\prime}}} \int_{Y_{\epsilon^{\prime}}} \int_{1 / k}^{b_{k-1}} \frac{d \lambda}{\left|\sum_{n=1}^{\infty}\left(i_{n}-j_{n}\right) \lambda^{n}\right|^{s}} d \mu_{\xi}(\underline{i}) d \mu \underline{\xi}(\underline{j}) d \bar{\mu}(\xi) \\
& =\int_{X_{\epsilon^{\prime}}} \int_{Y_{\epsilon^{\prime}}} \int_{X_{\epsilon^{\prime}}} \int_{1 / k}^{b_{k-1}} \frac{d \lambda}{e^{(h(\mu \mid \mathcal{A})-2 \epsilon)|\underline{i} \wedge \underline{j}|}\left|\sum_{n=0}^{\infty} a_{n} \lambda^{n}\right|^{s}} d \mu_{\xi}(\underline{i}) d \mu \underline{\xi}(\underline{j}) d \bar{\mu}(\xi)
\end{aligned}
$$

where we denote

$$
|\underline{i} \wedge \underline{j}|=\min \left\{l: i_{s}=j_{s}, 0 \leq s \leq l\right\},
$$

and we have that $a_{n} \in\{0, \pm 1, \ldots, \pm(k-1)\}$ and $a_{0} \neq 0$. Thus we can use transversality (Proposition 2.1) to write

$$
\begin{aligned}
I & \leq C \int_{X_{\epsilon^{\prime}}} \int_{Y_{\epsilon^{\prime}}} \int_{Y_{\epsilon^{\prime}}} e^{-(h(\mu \mid \mathcal{A})+2 \epsilon) \underline{i} \wedge \underline{j}} d \mu_{\xi}(\underline{i}) d \mu_{\xi}(\underline{j}) d \bar{\mu}(\xi) \\
& \leq C \sum_{m=0}^{\infty} e^{-m(h(\mu \mid \mathcal{A})+2 \epsilon)}\left(\mu_{\xi} \times \mu_{\xi}\right)\left(\left\{(\underline{i}, \underline{j}) \in Y_{\epsilon^{\prime}} \times Y_{\epsilon^{\prime}}: i_{a}=j_{b}, 0 \leq a \leq m\right\}\right) \\
& \leq C K \sum_{m=0}^{\infty} e^{-m(h(\mu \mid \mathcal{A})+2 \epsilon)} e^{(h(\mu \mid \mathcal{A})+\epsilon) m}<+\infty .
\end{aligned}
$$

In particular, from the finiteness of (4.2) we deduce that for almost every $\lambda \in\left[1 / k, b_{k-1}\right]$, there is a set $Y=Y(\lambda) \subset \Pi_{\lambda}(X)$ of $\bar{v}$ measure $1-\epsilon^{\prime}$ such that for $y \in Y$ one can choose $\xi \in \bar{\Pi}_{\lambda}^{1}(y)$ such that

$$
\int_{\Pi_{\lambda} Y_{\epsilon^{\prime}}} \int_{\Pi_{\lambda} Y_{\epsilon^{\prime}}} \frac{d \nu_{\xi, \lambda}(x) d \nu_{\xi, \lambda}(y)}{|x-y|^{s}}<+\infty
$$


The mass distribution principle shows that $\operatorname{dim}_{H}\left(\nu_{\xi, \lambda}\right) \geq s$. Finally, since $\epsilon>0$ was arbitrary, the result follows.

The following corollary will prove particularly useful.

COROllary.

$$
\operatorname{dim}_{H}\left(v_{\lambda}\right) \geq \operatorname{dim}_{H}\left(\bar{v}_{\lambda}\right)-\min \left\{\frac{h(\mu \mid \mathcal{A})}{\log \lambda}, 1\right\} .
$$

Proof. Fix $\epsilon>0$. We can choose $X \subset \Lambda_{\lambda}$ with $\nu_{\lambda}(X)=1$ and $\operatorname{dim}_{H}(X)<\operatorname{dim}_{H}\left(\nu_{\lambda}\right)+\epsilon$. Using a variant on the Marstrand slice theorem (cf. $[5,2])$ we can bound

$$
\begin{aligned}
\operatorname{dim}_{H}(v)+\epsilon & \geq \operatorname{dim}_{H}(X) \\
& \geq \operatorname{dim}_{H}(Y)-\min \left\{\frac{h(\mu \mid \mathcal{A})}{\log \lambda}, 1\right\} \\
& \geq \operatorname{dim}_{H}\left(\bar{v}_{\lambda}\right)-\min \left\{\frac{h(\mu \mid \mathcal{A})}{\log \lambda}, 1\right\} .
\end{aligned}
$$

Since $\epsilon>0$ is arbitrary, the result follows.

Proof of Theorem 3. The inequality $\operatorname{dim}_{H}\left(\nu_{\lambda}\right) \leq-h(\mu) / \log \lambda$ for $(2.1)$ is easily seen to hold for all $0<\lambda<1$. More precisely, by the Shannon-McMillan-Brieman theorem we have that

$$
h(\mu)=\lim _{N \rightarrow+\infty} \frac{1}{N} \log \mu\left(\left[x_{0}, \ldots, x_{N-1}\right]\right)
$$

for a.e. $(\mu) x \in \Sigma_{n}$, where $\left[x_{0}, \ldots, x_{N-1}\right]=\left\{y \in \Sigma_{n}: y_{j}=x_{j}, 0 \leq j \leq N-1\right\}$. In particular,

$$
\limsup _{N \rightarrow+\infty} \frac{\log \nu_{\lambda}\left(B\left(x, \lambda^{N}\right)\right)}{\log \lambda^{N}} \leq-\frac{h(\mu)}{\log \lambda}
$$

gives a bound on the pointwise dimension, and thus for the Hausdorff dimension.

To get the reverse inequality, one can compare Proposition 2.2, Proposition 3.2 and the above corollary. This completes the proof of Theorem 3 .

Proof of Theorem 1. In particular, if we let $\mu=(1 / n, \ldots, 1 / n)^{\mathbb{Z}^{+}}$be the standard Bernoulli measure then $h(\mu)=\log n$. In particular, Theorem 1 follows from Theorem 3 .

5. Sets of positive measure, projections and transversality

We recall that a measure $v_{\lambda}$ on $\mathbb{R}^{d}$ is absolutely continuous if for any Borel set $A \subset \mathbb{R}^{d}$ satisfying $\operatorname{leb}(A)=0$ necessarily also satisfies $v_{\lambda}(A)=0$. The absolute continuity of the measure $\bar{\nu}_{\lambda}$ (where $d=1$ ) has been studied by Simon et al [18], who showed the following nice result.

Proposition 5.1. (Simon, Solomyak and Urbanski) For almost all $\lambda$ in the set

$$
\left\{\left[\frac{1}{k}, b_{k-1}\right]: h(\bar{\mu}) \geq-\log \lambda\right\},
$$

the measure $\bar{v}_{\lambda}=\bar{\Pi}_{\lambda} \bar{\mu}$ is absolutely continuous. 
A key ingredient in the proof of Proposition 5.1 is the following application of the transversality technique.

Lemma 5.2. [11] Let $\xi \in \Sigma_{k}$. There exists $C>0$ such that if $\underline{i}, \underline{j} \in p^{-1}(\xi)$ then for $a>0$

$$
\operatorname{leb}\left\{\lambda \in\left(a, b_{k-1}\right):\left|\bar{\Pi}_{\lambda}(\underline{i})-\bar{\Pi}_{\lambda}(\underline{j})\right| \leq \epsilon\right\} \leq C\left(a^{-|\underline{i} \wedge \underline{j}|}\right) \epsilon .
$$

The following result should be viewed as a two-dimensional version of Proposition 5.1.

THEOREM 4. For almost all $\lambda$ in the set

$$
\left\{\left[\frac{1}{k}, b_{k-1}\right]: \min \{h(\mu \mid \mathcal{A}), h(\bar{\mu})\} \geq-\log \lambda\right\},
$$

the measure $v_{\lambda}=\Pi_{\lambda} \mu$ is absolutely continuous.

For such $\lambda$ the set $\Lambda_{\lambda}$ has positive Lebesgue measure. In particular, Theorem 2 then follows from Theorem 4 .

A key ingredient in the proof of Theorem 4 is that typical measures $\nu_{\xi}$ are absolutely continuous on $L \bar{\Pi}_{\lambda}(\xi)$. This is contained in the following result.

Proposition 5.3. For almost all $\lambda$ in

$$
\left\{\lambda \in\left[\frac{1}{k}, b_{k-1}\right]: h(\mu \mid \mathcal{A})>-\log \lambda\right\},
$$

there exists a set $X \subseteq \Sigma_{k}$ such that $\bar{\mu}(X)=1$ and for any $\xi \in X$ the measure $v_{\lambda, \epsilon}$ is absolutely continuous on $L \bar{\Pi}_{\lambda}(\xi)$.

Proof. It suffices to show that, given $\epsilon^{\prime}>0$, there exists a set $X_{\epsilon^{\prime}} \subseteq \Sigma_{k}$ such that $\bar{\mu}\left(X_{\epsilon^{\prime}}\right) \geq 1-\epsilon^{\prime}$ and for any $\xi \in X_{\epsilon^{\prime}}$ there exists a set $Y_{\epsilon^{\prime}, \xi} \subset L_{\bar{\Pi}_{\lambda}(\xi)}$ where $\mu_{\xi}\left(Y_{\epsilon}^{\prime}\right) \geq 1-\epsilon^{\prime}$ and $\nu_{\lambda, \epsilon}$ is absolutely continuous on $Y_{\epsilon^{\prime}, \xi}$. We can then take $X=\bigcap_{n=1}^{\infty} X_{1 / n}$.

Let $\epsilon, \epsilon^{\prime}>0$. From Proposition 3.1 we know that there exists $K>0$ and a set $X_{\epsilon^{\prime}} \subseteq \Sigma_{k}$ such that $\bar{\mu}\left(X_{\epsilon^{\prime}}\right)>1-\epsilon^{\prime}$ and for $\xi \in X_{\epsilon^{\prime}}$ there exists $Y_{\epsilon^{\prime}, \xi} \subseteq p^{-1} \xi$ with $\mu_{\xi}\left(Y_{\epsilon^{\prime}, \xi}\right)>1-\epsilon^{\prime}$ and for $\underline{x} \in Y_{\epsilon^{\prime}, \xi}$ equation (3.1) holds, i.e.,

$$
\mu_{\xi}\left[x_{0}, \ldots, x_{N-1}\right] \leq K \exp (-(h(\mu \mid \mathcal{A})-\epsilon) N), \quad \text { for } N \geq 1 .
$$

We recall that the lower pointwise density for $\nu_{\lambda, \xi}$ (restricted to $\Pi_{\lambda} Y_{\epsilon^{\prime}, \xi}$ ) is defined by

$$
\underline{D}\left(v_{\xi}\right)(x)=\liminf _{\epsilon \searrow 0} \frac{\nu_{\xi}\left(B(x, \epsilon) \cap \Pi_{\lambda} Y_{\epsilon^{\prime}, \xi}\right)}{2 \epsilon} .
$$

To show that $\nu_{\xi}$ is absolutely continuous it suffices to show that $\underline{D}\left(v_{\xi}\right)(x)$ is finite, for a.e. $\left(v_{\xi, \lambda}\right) x \in \Pi_{\lambda} Y_{\epsilon^{\prime}, \xi}$. In particular, it suffices to show that

$$
\int_{\Pi_{\lambda} Y_{\epsilon^{\prime}, \xi}} \underline{D}\left(v_{\xi, \lambda}\right)(x) d v_{\xi, \lambda}(x)<+\infty .
$$

Moreover, to show that for almost every $\lambda$ there exists a set of $\xi$ of $\bar{\mu}$ measure at least $1-\epsilon^{\prime}$ such that $\nu_{\xi, \lambda}$ is absolutely continuous, it suffices to show that

$$
I:=\int_{t}^{b_{k-1}} \int_{X_{\epsilon^{\prime}}}\left(\int_{\Pi_{\lambda} Y_{\epsilon^{\prime}, \xi}} \underline{D}\left(\nu_{\xi, \lambda}\right)(x) d \nu_{\xi, \lambda}(x)\right) d \bar{\mu}(\xi) d \lambda<+\infty,
$$


providing $t$ is sufficiently large. We take $t>e^{h(\mu \mid \mathcal{A})+2 \epsilon}$. For $\omega, \tau \in p^{-1} \xi$ we define

$$
\phi_{r}(\omega, \tau)=\left\{\lambda:\left|\Pi_{\lambda}(\omega)-\Pi_{\lambda}(\tau)\right| \leq r\right\},
$$

for $r>0$. We start by lifting to the shift space, applying Fatou's lemma and Fubini's theorem

$$
\begin{aligned}
I & \leq \liminf _{r \rightarrow 0} \frac{1}{2 r} \int_{t}^{b_{k-1}} \int_{X_{\epsilon^{\prime}}} \int_{Y_{\epsilon^{\prime}, \xi}} \int_{Y_{\epsilon^{\prime}, \xi}} \chi(\omega, \tau) \omega, \tau \mu_{\xi}(\omega) d \mu_{\xi}(\tau) d \bar{\mu}(\xi) d \lambda \\
& \leq \liminf _{r \rightarrow 0} \frac{1}{2 r} \int_{X_{\epsilon^{\prime}}} \int_{Y_{\epsilon^{\prime}, \xi}} \int_{Y_{\epsilon^{\prime}, \xi}} \operatorname{leb}\left(\phi_{r}(\omega, \tau)\right) d \mu_{\xi}(\omega) d \mu_{\xi}(\tau) d \bar{\mu}(\xi),
\end{aligned}
$$

where $\chi$ is the characteristic function for $\left\{(\omega, \tau):\left|\Pi_{\lambda}(\omega)-\Pi_{\lambda}(\tau)\right| \leq r\right\}$. We can apply Lemma 5.2 and then use equation (5.1):

$$
\begin{aligned}
I & \leq C \int_{X_{\epsilon^{\prime}}} \int_{Y_{\epsilon^{\prime}, \xi}} \int_{Y_{\epsilon^{\prime}, \xi}} t^{-|\omega \wedge \tau|} d \mu_{\xi}(\omega) d \mu_{\xi}(\tau) d \bar{\mu}(\xi) \\
& \leq C \int_{X_{\epsilon^{\prime}}} \int_{Y_{\epsilon^{\prime}, \xi}} \int_{Y_{\epsilon^{\prime}, \xi}} e^{-|\omega \wedge \tau|(h(\mu \mid \mathcal{A})+2 \epsilon)} d \mu_{\xi}(\omega) d \mu_{\xi}(\tau) d \bar{\mu}(\xi) \\
& \leq C \int_{X_{\epsilon^{\prime}}} \sum_{m=0}^{\infty} e^{-m(h(\mu \mid \mathcal{A})+2 \epsilon)}\left(\mu_{\xi} \times \mu_{\xi}\right)\left(\Delta_{m}\right) d \bar{\mu}(\xi) \\
& \leq C K \sum_{m=0}^{\infty} e^{-m(h(\mu \mid \mathcal{A})+2 \epsilon)} e^{m(h(\mu \mid \mathcal{A})+\epsilon)}<\infty,
\end{aligned}
$$

where $\Delta_{m}=\left\{(\tau, \omega) \in Y_{\epsilon^{\prime}, \xi} \times Y_{\epsilon^{\prime}, \xi}: \omega_{1}=\tau_{1}, \ldots, \omega_{m}=\tau_{m}\right\}$. This completes the proof.

Proof of Theorem 4. By Proposition 5.1 and Lemma 5.2 it follows that $\bar{v}_{\lambda}$ is absolutely continuous and there exists a set $X_{\lambda} \subseteq \Sigma_{k}$ such that for all $\xi \in X_{\lambda}, \nu_{\xi, \lambda}$ is absolutely continuous on $L_{\Pi_{\lambda}(\xi)}$ for almost all $\lambda$. We choose $\lambda$ to satisfy these properties. Let $Y \subseteq \mathbb{R}^{2}$ be a set such that $\operatorname{leb}^{2}(Y)=0$. Let $G=\left\{y \in \mathbb{R}: \operatorname{leb}\left(L_{y}\right)=0\right\}$, then it is clear that $\operatorname{leb}(G)=0$. Using the decomposition of $\mu$,

$$
\begin{aligned}
v_{\lambda}(Y) & =\mu\left(\Pi_{\lambda}^{-1}(Y)\right)=\int \mu_{\xi}\left(\Pi_{\lambda}^{-1}(Y) \cap p^{-1} \xi\right) d \bar{\mu}(\xi) \\
& =\int_{X_{\lambda}} \mu_{\xi}\left(\Pi_{\lambda}^{-1}(Y) \cap p^{-1} \xi\right) d \bar{\mu}(\xi) .
\end{aligned}
$$

Let $\Gamma=\left\{\xi \in X_{\lambda}: \mu_{\xi}\left(\Pi_{\lambda}^{-1}(Y) \cap p^{-1} \xi\right)>0\right\}$. If $\xi \in \Gamma$ then $v_{\lambda, \xi}\left(Y \cap L_{\Pi_{\lambda} \xi}\right)>0$ and hence $\operatorname{leb}\left(Y \cap L_{\Pi_{\lambda} \xi}\right)>0$. Thus if $\xi \in \Gamma$ then $\bar{\Pi}_{\lambda} \xi \in G$. From the absolute continuity of $\bar{v}_{\lambda}$ it follows that $\bar{\nu}_{\lambda}(G)=0$ and so $\bar{\mu}(\Gamma)=0$. Hence $\nu_{\lambda}(Y)=0$ and it follows that $\nu_{\lambda}$ is absolutely continuous.

Remark 1. In order to derive the bound in (0.3), we can consider the Bernoulli measure $\bar{\mu}=\left(q_{1}, \ldots, q_{k}\right)^{\mathbb{Z}^{+}}$on $\Sigma_{k}$. Let $\mu=\left(q_{1} / n_{1}, \ldots, q_{k} / n_{k}\right)^{\mathbb{Z}^{+}}$be the Bernoulli measure on $\Sigma_{n}$. We have that $h(\bar{\mu})=-\sum_{j=1}^{k} q_{j} \log q_{j}$ and $h(\mu)=-\sum_{j=1}^{k} q_{j} \log \left(q_{j} / n_{j}\right)$. In particular, we see that

$$
h(\mu \mid \mathcal{A})=h(\mu)-h(\bar{\mu})=\sum_{j=1}^{k} q_{j} \log n_{j} .
$$


For any $\left(q_{1}, \ldots, q_{k}\right)$ such that $h(\bar{\mu})=h(\mu \mid \mathcal{A})$ we could might choose $t=e^{-h(\bar{\mu})}$ in Theorem 3 .

Remark 2. If we consider $\mu$ supported on some subshift $\Sigma$ on $k$-symbols then there is a possibility that the transversality constant can be increased. This was considered by Solomyak [19]. For example, by recoding by words of length 3 we can restrict to symbols of the form $[*, *, 0]$ to get a subshift $\widehat{\sigma}: \Sigma_{A} \rightarrow \Sigma_{A}$. This reduces the entropy to $h(\widehat{\sigma})=\frac{2}{3} h(\sigma)$. However, the advantage is that the transversality constant $b_{k-1}$ is also increased, to $\widehat{b}_{k-1}>\frac{2}{3}$ (see [19]). This technique allows us to extend the absolute continuity results to larger domains of $\lambda$.

\section{Comments and generalizations}

6.1. Limitations on the estimates. It is easy to construct examples for which one can find an open interval $U \subset[1 / k, 1 / \sqrt{n}]$ for which $\operatorname{dim}_{H}\left(\Lambda_{\lambda}\right)<-\log n / \log \lambda$ for $\lambda \in U$.

Example 4. Let $k=3$ and let $c_{1}, \ldots, c_{5}$ correspond to the four corners, plus $(1,0)$ square. In this case, $s=3^{-3} * 2^{-2} / 5=0.3920 \ldots$ However for $\lambda>0.4$ we have that $\operatorname{dim}_{\mathrm{H}}\left(\Lambda_{\lambda}\right) \leq 1-\log 2 / \log \lambda<-\log 5 / \log \lambda$. For $\lambda>0.4082 \ldots$ we let $\mu$ be the $\left(\frac{1}{6}, \frac{1}{6}, \frac{1}{6}, \frac{1}{4}, \frac{1}{4}\right)$-Bernoulli measure on $\Sigma_{5}$ and $\nu_{\lambda}$ the projection of $\mu$ onto $\Lambda_{\lambda}$. Theorem 3 gives $\operatorname{dim}_{H}\left(\nu_{\lambda}\right) \geq 1-\log 2 / \log \lambda$ for a.e. $\lambda>0.4082 \ldots$ and thus $\operatorname{dim}_{H}\left(\Lambda_{\lambda}\right)=$ $1-\log 2 / \log \lambda$ for a.e. $\lambda>0.4082 \ldots$

6.2. More general contractions. It is easy to see (using an affine transformation of the plane) that we can consider more general grids $\mathcal{C}$ by translating horizontally each row by the same amount. More generally, we can consider parametrized families contractions $T_{i j}^{(\lambda)}: \mathbb{R}^{2} \rightarrow \mathbb{R}^{2}$ of the plane given by

$$
T_{i j}:(x, y) \mapsto\left(f_{i}^{(\lambda)}(x), g_{i j}^{(\lambda)}(x, y)\right),
$$

where $f_{i}^{(\lambda)}:[0,1] \rightarrow[0,1]$ and $g_{i j}^{(\lambda)}:[0,1] \times[0,1] \rightarrow[0,1]$ are $C^{\infty}$ contractions. An important feature is that the foliation of the plane by vertical lines $\{x\} \times \mathbb{R}$, for $x \in \mathbb{R}$, is preserved under the maps, i.e. $T_{i j}^{(\lambda)} L_{x}=L_{f_{i}^{(\lambda)}(x)}$.

Let $\Sigma_{n}$ be the space of sequences with symbols $(i, j)$ and let $\sigma: \Sigma_{n} \rightarrow \Sigma_{n}$ be the full shift on $n$ symbols defined by $(\sigma x)_{n}=x_{n+1}$. Let $\Pi_{\lambda}: \Sigma_{n} \rightarrow \mathbb{R}^{2}$, where

$$
\Pi_{\lambda}(\underline{x})=\sum_{m=1}^{\infty} T_{x_{1}}^{(\lambda)} \cdots T_{x_{m}}^{(\lambda)}(0,0),
$$

say, is the natural map to the associated attractor $\Lambda_{\lambda}$. Let $\Sigma_{k}$ be the space of sequences with symbols $i$ and let $\sigma: \Sigma_{k} \rightarrow \Sigma_{k}$ be the coding corresponding to the iterated function scheme $\left\{f_{i}^{(\lambda)}\right\}_{i=1}^{k}$ and let $\bar{\Pi}_{\lambda}: \Sigma_{k} \rightarrow \bar{\Lambda}_{\lambda}$ be the associated map. Let $\mu$ be an ergodic probability measure on $\Sigma_{n}$ and let $\bar{\mu}$ be the corresponding ergodic probability measure on $\Sigma_{k}$. We decompose $\mu$ as in $\S 3$, for any Borel set $A \subseteq \Sigma_{n}, \mu(A)=\int_{\Sigma_{k}} \mu_{\xi}(A) d \bar{\mu}(\xi)$ and denote $\Pi_{\lambda}$ as the restriction of $\Pi_{\lambda}$ to $p^{-1} \xi$. Thus we can define measures $\nu_{\lambda}=\mu \circ \Pi_{\lambda}^{-1}$ 
on $\Lambda_{\lambda}, \bar{v}_{\lambda}=\bar{\mu} \circ \bar{\Pi}_{\lambda}^{1}$ on $\bar{\Lambda}_{\lambda}$ and $v_{\lambda, \xi}=\mu_{\xi} \circ \Pi_{\lambda}^{-1}$ on $L_{\bar{\Pi}_{\lambda} \xi}$, respectively. We can associate two Lyapunov exponents

$$
\chi_{1}=\int_{\Sigma_{k}} \log \left|f_{x_{0}}^{\prime}\right| \circ \bar{\Pi}_{\lambda} d \bar{\mu}(x) \quad \text { and } \quad \chi_{2}=\int_{\Sigma_{k}} \int_{p^{-1} \xi} \log \left|\frac{\partial g_{x_{0}}}{\partial y}\right| \circ \Pi_{\lambda} d \mu_{\xi}(\underline{x}) d \bar{\mu}(\xi) .
$$

We need the following transversality conditions to be satisfied. There exists a constant $C_{1}>0$ so that for $\omega, \tau \in \Sigma_{k}$ with $\omega_{0} \neq \tau_{0}$

$$
\operatorname{leb}\left\{\lambda \in U:\left|\bar{\Pi}_{\lambda}(\omega)-\bar{\Pi}_{\lambda}(\tau)\right| \leq r\right\} \leq C_{1} r
$$

and for any $\xi \in \Sigma_{k}$ there exists a constant $C_{2}$ so that for $\underline{\omega}, \underline{\tau} \in p^{-1} \xi$

$$
\operatorname{leb}\left\{\lambda \in U:\left|\Pi_{\lambda}(\omega)-\Pi_{\lambda}(\tau)\right| \leq r\right\} \leq C_{2} r .
$$

We let $A_{\lambda}$ be the set where both transversality conditions are satisfied. We can now state analogues to Theorem 3 .

Proposition 6.1. For almost all $\lambda \in A_{\lambda}$ :

$$
\begin{aligned}
& \operatorname{dim}_{H}\left(v_{\lambda}\right) \geq-\left(\frac{h(\bar{\mu})}{\chi_{1}}+\frac{h(\mu \mid \mathcal{A})}{\chi_{2}}\right) \quad \text { if } \max \left\{-\frac{h(\bar{\mu})}{\chi_{1}},-\frac{-h(\mu \mid \mathcal{A})}{\chi_{2}}\right\} \leq 1 ; \\
& \operatorname{dim}_{H}\left(v_{\lambda}\right) \geq 1+\min \left\{-\frac{-h(\bar{\mu})}{\chi_{1}},-\frac{h(\mu \mid \mathcal{A})}{\chi_{2}}\right\} \text { otherwise. }
\end{aligned}
$$

The analogue of Theorem 4 is the following.

Proposition 6.2. For almost all $\lambda$ in the set

$$
\left\{\lambda \in A_{\lambda}: \min \left\{-\frac{h(\bar{\mu})}{\chi_{1}},-\frac{h(\mu \mid \mathcal{A})}{\chi_{2}}\right\} \geq 1\right\}
$$

$v_{\lambda}$ is absolutely continuous.

Application. (Bedford-McMullen [10]) This setting includes the generalized Sierpinski carpet studied by McMullen and Bedford. Let $k . m \geq 2$ and write $\beta=\log k / \log m$. Consider contractions

$$
\begin{gathered}
T_{i}: \mathbb{R}^{2} \rightarrow \mathbb{R}^{2}, \\
T_{i}:(x, y) \mapsto\left(\lambda x, \lambda^{\beta} y\right)+c_{i},
\end{gathered}
$$

where $c_{i} \in\{(j, l): 0 \leq j \leq k-1,0 \leq l \leq m-1\}, i=1, \ldots, n$ are distinct points. Let $\Lambda_{\lambda, \lambda^{\beta}}$ be the associated limit set. In the particular case that $\lambda=1 / m$ and $\lambda^{\beta}=1 / k$ this corresponds to the generalized Sierpinski carpet construction of McMullen and Bedford. The same general method allows one to show there exists $s>1 / k$ such that for almost all $\lambda \in[1 / k, s]$ we have that

$$
\operatorname{dim}\left(\Lambda_{\lambda, \lambda^{\beta}}\right)=\frac{\log \left(\sum_{i=0}^{k-1} n_{i}^{\beta}\right)}{-\log \lambda}
$$

where $n_{i}=\operatorname{Card}\left\{c_{i}: c_{i}^{(1)}=i\right\}$. To see this is a lower bound let

$$
p_{i}=\frac{n_{i}^{\beta-1}}{\sum_{j=0}^{k-1} n_{i}^{\beta}}
$$


for $i=1, \ldots, n$ and $\mu$ be $\left(p_{1}, \ldots, p_{n}\right)$ Bernoulli measure on $\Sigma_{n}$. If we let $\nu_{\lambda}$ be the natural projection of $\mu$ onto $\Lambda_{\lambda, \lambda^{\beta}}$ then Proposition 6.1 can be used to show that there exists $s$ such that

$$
\operatorname{dim} v_{\lambda} \geq \frac{\log \left(\sum_{i=0}^{k-1} n_{i}^{\beta}\right)}{-\log \lambda}
$$

for almost every $\lambda \in[1 / k, s]$. A simple adjustment of McMullen's argument to account for the overlaps shows that this is also an upper bound.

In [12] Peres and Solomyak ask whether it is possible to find an example of a selfsimilar set with positive measure but empty interior (question 2.4). We cannot answer this question $\dagger$ but we can show that there exist simple examples of self-affine sets in $\mathbb{R}^{2}$ with positive measure and empty interior. Let

$$
T_{i}:(x, y) \rightarrow\left(\frac{1}{3} x, \lambda y\right)+c_{i}
$$

for $1 \leq i \leq 7$ and where $c_{1}=(0,0), c_{2}=(0,1), c_{3}=(0,2), c_{4}=(1,1), c_{5}=$ $(2,0), c_{6}=(2,1)$ and $c_{7}=(2,2)$. Let $\Lambda(\lambda)$ be the associated limit set. We can choose $\mu$ to be the Bernoulli measure defined by the probability vector $\left(\frac{1}{9}, \frac{1}{9}, \frac{1}{9}, \frac{1}{3}, \frac{1}{9}, \frac{1}{9}, \frac{1}{9}\right)$. Let $v_{\lambda}$ be the natural projection of $\mu$ onto $\Lambda(\lambda)$. In the setting of Propositions 6.1 and 6.2 we have $\chi_{1}=-\log 3, \chi_{2}=\log \lambda, h(\bar{\mu})=\log 3$ and $h(\mu \mid \mathcal{A})=0.7324 \ldots$ Thus,

$$
\min \left\{-\frac{h(\bar{\mu})}{\chi_{1}},-\frac{h(\mu \mid(A))}{\chi_{2}}\right\} \geq 1 \quad \text { for } \lambda>0.4807 \ldots
$$

Since for $\lambda<\frac{1}{2}$ the transversality conditions hold, we have by the method of Proposition 6.2 that for almost all $\lambda \in(0.4807 \ldots, 0.5)$ the measure $v_{\lambda}$ is absolutely continuous, and hence $\Lambda(\lambda)$ has positive measure.

We now need to show that $\Lambda(\lambda)$ has empty interior. Note that $\Sigma_{k}=\{0,1,2\}^{\mathbb{N}}$ and $\bar{\Pi}_{\lambda}(\underline{x})=\sum_{n=0}^{\infty} x_{n}\left(\frac{1}{3}\right)^{n}$. Consider the set

$$
A=\left\{x \in \mathbb{R}: \exists \underline{x} \in\{0,1,2\}^{\mathbb{N}}, N \in \mathbb{N} \text { such that } \bar{\Pi}_{\lambda}(\underline{x})=x \text { and } \forall n \geq N, x_{n}=1\right\} .
$$

This set is clearly dense in $\overline{\Lambda(\lambda)}=[0,3]$ and for any $x \in A$ the sequence $x \in\{0,1,2\}^{\mathbb{N}}$ such that $\bar{\Pi}_{\lambda}(\underline{x})=x$ is unique. Given $x \in A$, let $L_{x}$ be the vertical line intersecting $(x, 0)$. If $\underline{y} \in \Sigma_{n}$ and $\Pi_{\lambda}(\underline{y}) \in L_{x}$ then whenever $x_{n}=1$ necessarily $y_{n}=(1,1)$. However, by hypothesis $x_{n}=1$ for all $n \geq N$ and thus there are only a finite number of sequences $\underline{y}$ such that $\Pi_{\lambda}(\underline{y}) \in L_{x}$. Hence $L_{x} \cap \Lambda(\lambda)$ contains a finite number of points for any given $x \in A$. Since $\bar{A}$ is dense in $\overline{\Lambda(\lambda)}=[0,3]$ the set $\Lambda(\lambda)$ cannot contain open sets and so has empty interior.

Remark. Most of the elements of the above proofs depend on entropy and are essentially measure theoretic in flavour. Thus, it is possible to extend many of these arguments to nonuniformly hyperbolic systems (e.g., parabolic points, systems which contract in mean).

$\dagger$ Added in proof: This question has now been answered in the appendix to this paper [21] 
6.3. Higher dimensions. There are natural extensions to higher dimensions. Perhaps this is best illustrated by simple examples in $\mathbb{R}^{3}$.

Example 1. Consider the Menger sponge, consisting of 20 contractions. We can associate to the corresponding subshift $\Sigma_{20}$ the Bernoulli measure $\mu$ with equal weights $1 / 20$. The sponge projects to the Sierpinski gasket, and the measure $\mu$ projects to a Bernoulli measure $\bar{\mu}$ on $\Sigma_{8}$ given by

$$
\bar{\mu}=\left(\frac{1}{10}, \frac{1}{10}, \frac{1}{10}, \frac{1}{10}, \frac{3}{20}, \frac{3}{20}, \frac{3}{20}, \frac{3}{20}\right)^{\mathbb{N}} .
$$

Finally, this projects to a Bernoulli measure $\widehat{\mu}$ on $\mathbb{R}$ given by $\widehat{\mu}=\left(\frac{2}{5}, \frac{1}{5}, \frac{2}{5}\right)^{\mathbb{N}}$. The entropies are $h(\mu)=\log (20)=2.9957, h(\bar{\mu})=\log (5)-\frac{1}{2} \log (2)-\frac{1}{2} \log (3)=$ $2.099 \ldots$ and $h(\widehat{\mu})=\log (5)-\frac{4}{5} \log (2)=1.054 \ldots$ The method we described before applies providing

$$
\lambda \leq \min \left\{e^{-(h(\mu)-h(\bar{\mu}))}, e^{-(h(\bar{\mu})-h(\widehat{\mu}))}, e^{-h(\widehat{\mu})}\right\}=0.348 \ldots .
$$

Consider the probability vector $\left(q_{1}, q_{2}, q_{3}\right)$ for $\bar{\mu}$ as in Example 2 of the Introduction. If we choose the probability vector for the 20 subsquares with weights $q_{1} / 8$ and $q_{2} / 4$ then we see that the measure is absolutely provided $\lambda \geq 0.393 \ldots$.

Example 2. Consider a Sierpinski tetrahedron, consisting of four contractions. We can associate to the corresponding subshift $\Sigma_{4}$ the Bernoulli measure $\mu=\left(\frac{1}{4}, \frac{1}{4}, \frac{1}{4}, \frac{1}{4}\right)^{\mathbb{N}}$. The sponge projects to the Sierpinski gasket, and the measure $\mu$ projects to a Bernoulli measure $\bar{\mu}$ on $\Sigma_{8}$ given by $\bar{\mu}=\left(\frac{1}{4}, \frac{1}{2}, \frac{1}{4}\right)$. Finally, this projects to a Bernoulli measure $\widehat{\mu}$ on $\mathbb{R}$ given by $\widehat{\mu}=\left(\frac{1}{4}, \frac{3}{4}\right)^{\mathbb{N}}$. The entropies are $h(\mu)=\log (4), h(\bar{\mu})=\log (2)$ and $h(\widehat{\mu})=\log (4)-\log (3)$. The method we described before applies providing

$$
\lambda \leq \min \left\{e^{-(h(\mu)-h(\bar{\mu}))}, e^{-(h(\bar{\mu})-h(\widehat{\mu}))}, e^{-h(\widehat{\mu})}\right\}=0.569 \ldots
$$

Acknowledgements. The diagrams were drawn using Matlab and the numerical calculations done using 'Mathematica'. We would like to thank Nikita Sidorov for useful conversations.

\section{REFERENCES}

[1] L. M. Abramov and V. A. Rohlin. The entropy of a skew product of measure preserving transformations. Trans. Amer. Math. Soc. (2) 48 (1966), 255-265.

[2] C. Bishop. Topics in real analysis (unpublished lecture notes) http://www.math.sunysb.edu/ bishop/classes/math639.S01/math639.html

[3] T. Bogenschütz and H. Crauel. The Abramov-Rokhlin Formula (Lecture Notes in Mathematics, 1514). Springer, Berlin, 1992, 32-35.

[4] D. Broomhead, J. Montaldi and N. Sidorov. Golden gaskets: variations on the Sierpinski sieve. Preprint. Available at: http://www.ma.umist.edu/ nikita/gold-final.pdf

[5] K. Falconer. Fractal Geometry. Wiley, London, 1990.

[6] L. Jonker and J. Veerman. Semi-continuity of dimension and measure of locally scaled fractals. Fund. Math. 173 (2002), 113-131. 
[7] T. Jordan. Dimension of Fat Sierpinski gaskets. Preprint. Available at: http://www.maths.warwick.ac.uk/ tjordan/overlapgasket.pdf.

[8] F. Ledrappier and L.-S. Young. The metric entropy of diffeomorphisms. II. Relations between entropy, exponents and dimension. Ann. of Math. (2) 112 (1985), 540-574.

[9] F. Ledrappier and P. Walters. A relativised variational principle for continuous transformations. J. London. Math. Soc. 16 (1977), 568-576.

[10] C. McMullen. The Hausdorff dimension of general Sierpinski carpets. Nagoya Math. J. 96 (1984), 1-9.

[11] Y. Peres and B. Solomyak. Self-similar measures and the intersection of Cantor sets. Trans. Amer. Math. Soc. 350 (1998), 4065-4087.

[12] Y. Peres and B. Solomyak. Problems on self-similar and self-affine sets; an update. Progr. Probab. 46 (2000), 95-106.

[13] K. Petersen. Ergodic Theory. Cambridge University Press, Cambridge, 1983.

[14] M. Pollicott and K. Simon. The Hausdorff dimension of $\lambda$-expansions with deleted digits. Trans. Amer. Math. Soc. 347 (1995), 967-983.

[15] V. Rohlin. Lectures on the entropy theory of measure preserving transformations. Russian Math. Surveys 22(5) (1967), 1-52.

[16] V. Rohlin. On the fundamental ideas of measure theory. Trans. Amer. Math. Soc. 71 (1952), 1-54.

[17] K. Simon and B. Solomyak. On the dimension of self-similar sets. Fractals 10 (2003), 59-65.

[18] K. Simon, B. Solomyak and M. Urbanski. Invariant measures for parabolic IFS with overlaps and random continued fractions. Trans. Amer. Math. Soc. 353 (2001), 5145-5164.

[19] B. Solomyak. On the random series $\sum \pm \lambda^{n}$ (an Erdös problem). Ann. of Math. (2) 142 (1995), 611-625.

[20] P. Walters. Ergodic Theory. Springer, Berlin, 1982.

[21] M. Csörnyei, T. Jordan, M. Pollicott, D. Preiss and B. Solomyak. Positive-measure self-similar sets without interior, this issue. 